\title{
Upregulation of Yes-associated protein and transcriptional co-activator with PDZ-binding motif influences the behavior of LOVO human colon adenocarcinoma cells
}

\author{
YU LI, LAN LI, MIN ZHU, LIMIN YE and QIAN YANG \\ Department of Gastroenterology, Guizhou Provincial People's Hospital, Guiyang, Guizhou 550002, P.R. China
}

Received September 23, 2016; Accepted May 19, 2017

DOI: $10.3892 /$ etm.2017.4962

\begin{abstract}
The present study aimed to investigate the role of Yes-associated protein (YAP) and transcriptional co-activator with PDZ-binding motif (TAZ) in the LOVO human colon adenocarcinoma cell line and explore the underlying mechanisms. First, the expression levels of YAP and TAZ were detected in LOVO cells using reverse-transcription quantitative PCR, and the results suggested that YAP and TAZ were faintly expressed in LOVO cells. To investigate the exact role of YAP and TAZ in LOVO cells, stable YAP- and/or TAZ-overexpressing LOVO cell lines were established using YAP and/or TAZ expression plasmids. An MTT assay and flow cytometry were used to assess cell proliferation and apoptosis, respectively. The results indicated that compared with the control, YAP or TAZ overexpression significantly increased the proliferation ability of LOVO cells, while apoptosis was significantly decreased. Furthermore, the expression of the tumor-associated proteins connective tissue growth factor and cysteine-rich angiogenic inducer 61, which have critical roles in facilitating cancer cell proliferation, migration and invasion, were found to be upregulated following upregulation of YAP and TAZ. In addition, the expression of cell apoptosis-associated protein B-cell lymphoma 2 (Bcl-2) was significantly increased, while Bcl-2-associated $\mathrm{X}$ protein and caspase- 3 were inhibited by YAP or TAZ overexpression. All of these effects were amplified when YAP and TAZ were co-overexpressed. In conclusion, YAP and TAZ function as tumor promoters in human colon carcinoma, and upregulation of YAP and TAZ influences the behavior of LOVO colon adenocarcinoma cells via regulating tumor-associated gene expression.
\end{abstract}

\section{Introduction}

Colorectal carcinoma (CRC) is the second most common type of malignant disease in women and the third most common

Correspondence to: Dr Yu Li, Department of Gastroenterology, Guizhou Provincial People's Hospital, 83 East Zhongshan Road, Guiyang, Guizhou 550002, P.R. China

E-mail: liyu160907@163.com

Key words: human colorectal carcinoma, YAP, TAZ, proliferation, apoptosis type in men in the world and is associated with a high mortality and morbidity rate in developed countries (1). Surgery is an effective method for the treatment of CRC (2). Neoadjuvant therapy only provides a slight benefit for high-risk CRC patients, and due to the high cost and side effects, is not a satisfactory option (3). Thus, it is important to explore novel therapeutic strategies for CRC treatment.

The Hippo signaling pathway has crucial roles in cell proliferation regulation, organ size control and cancer progression (4-7). Yes-associated protein (YAP) and transcriptional co-activator with PDZ-binding motif (TAZ) are transcriptional co-activators, which are inhibited by Hippo pathway activation. Suppression of the Hippo signaling pathway leads the dephosphorylation of YAP and TAZ, resulting in nuclear translocation and induction of gene transcription. Studies have demonstrated that YAP was overexpressed in human colon cancer specimens and cell lines. Overexpression of YAP promoted the growth and survival of colon cancer cells, which was indicative of its oncogenic function and suggested that its overexpression is a substantial contributor in cancer development (8-10). In addition, studies have confirmed that TAZ is upregulated in CRC and has an important role in CRC progression via promoting cell proliferation and survival (11-13). As YAP and TAZ are involved in the development of CRC, their roles and the function of the Hippo signaling pathway in human $\mathrm{CRC}$ may be further explored with the aim of identifying novel treatment targets or molecular markers.

A previous study suggested that overexpression of YAP and TAZ is an independent prognostic factor in CRC, and that YAP and TAZ expression in LOVO human colon adenocarcinoma cells was low (13). However, the exact role of YAP and TAZ in LOVO cells and the underlying molecular mechanisms have remained to be fully elucidated. Thus, the present study investigated the role of YAP and TAZ in LOVO human colon adenocarcinoma cells and explored the underlying mechanisms.

\section{Materials and methods}

Cell culture. The LOVO and SW480 human colon adenocarcinoma cell lines were purchased from the Type Culture Collection of the Chinese Academy of Sciences (Shanghai, China). LOVO cells were cultured in Dulbecco's modified Eagle's medium (Hyclone; GE Healthcare, Little Chalfont, 
UK) supplemented with $10 \%$ fetal bovine serum (FBS; Hyclone; GE Healthcare), $1 \%$ penicillin and streptomycin in a humidified incubator with $95 \%$ air and $5 \% \mathrm{CO}_{2}$ at $37^{\circ} \mathrm{C}$. SW480 cells were cultured in RPMI 1640 medium (Gibco; Thermo Fisher Scientific, Inc., Waltham, MA, USA) with $10 \%$ FBS (Hyclone; GE Healthcare). Cells were passaged upon reaching $90 \%$ confluence.

Cell transfection. To investigate the role of YAP and TAZ in LOVO cells, YAP plasmids (product no. sc-400040-ACT; Santa Cruz Biotechnology, Inc., Dallas, TX, USA) and/or TZA plasmids (product no. sc-400320-ACT; Santa Cruz Biotechnology, Inc.) were transfected into LOVO cells with $30 \mu 1$ Lipofectamine 2000 transfection reagent (Invitrogen; Thermo Fisher Scientific, Inc.) following the manufacturer's instructions. At $24 \mathrm{~h}$ after transfection, the cells were used for the subsequent experiments.

Reverse-transcription quantitative polymerase chain reaction (RT-qPCR). Total RNA was extracted from LOVO cells using TRIzol reagent (Taraka, Otsu, Japan) following the manufacturer's instructions. The mRNA expression was determined using RT-qPCR. RT reactions were performed using PrimeScript ${ }^{\mathrm{TM}}$ RT reagent kit (Taraka). For real-time PCR, the SYBR $^{\circledR}$ Premix Ex Taq ${ }^{\mathrm{TM}}$ II (Taraka) was used, and all reactions were repeated three times with the following conditions: $95^{\circ} \mathrm{C}$ for $10 \mathrm{~min}$, followed by 38 cycles at $95^{\circ} \mathrm{C}$ for $15 \mathrm{sec}$ and $72^{\circ} \mathrm{C}$ for $30 \mathrm{sec}$. GAPDH was used as an internal control. The $2^{-\triangle \Delta C q}$ method (14) was used to determine the relative amounts of each transcript. The primers used for real time-PCR are listed in Table I.

Western blot analysis. Whole-cell protein was extracted using radioimmunoprecipitation assay buffer (SolarBio, Beijing, China), and protein samples of equal concentrations were prepared by standardization with a Nano Drop Microvolume Spectrophotometer (Thermo Scientific ${ }^{\mathrm{TM}}$ NannoDrop 2000) according to the manufacturer's instructions. A total of $25 \mu \mathrm{g}$ of protein samples were separated by $10 \%$ SDS-PAGE and transferred onto polyvinylidene difluoride membranes (Millipore, Billerica, MA, USA). Membranes were blocked at room temperature for $1 \mathrm{~h}$ in PBS solution supplemented with $0.1 \%$ Tween-20 and 5\% fat-free powdered milk, and blotted with primary antibodies to connective tissue growth factor (CTGF; sc-101586; Santa Cruz Biotechnology, Inc.), cysteine-rich angiogenic inducer 61 (Cyr61; sc-374129; Santa Cruz Biotechnology, Inc.), B-cell lymphoma 2 (Bcl-2; AB112; Beyotime Institute of Biotechnology, Shanghai, China), Bcl-2-associated X protein (Bax; AB026; Beyotime Institute of Biotechnology), caspase-3 (AC030; Beyotime Institute of Biotechnology) (all used at 1:1,000 dilution) and GAPDH (AG019; Beyotime Institute of Biotechnology; 1:2,000 dilution) at $4^{\circ} \mathrm{C}$ overnight, followed by horseradish peroxidase (HRP)-conjugated rabbit anti-mouse IgG antibody (A0192; Beyotime Institute of Biotechnology; 1:5,000 dilution) at room temperature for $2 \mathrm{~h}$. Signals were detected by the enhanced chemiluminescence method (Thermo Fisher Scientific, Inc.). The ChemiDoc ${ }^{\mathrm{TM}}$ XRS+ imaging system (Bio-Rad Laboratories, Inc., Hercules, CA, USA) was used to analyze the protein bands.
Table I. Primer sequences for polymerase chain reaction.

\begin{tabular}{lc}
\hline Gene/direction & \multicolumn{1}{c}{ Sequence (5'-3') } \\
\hline YAP & \\
Forward & ACGTTCATCTGGGACAGCAT \\
Reverse & GTTGGGAGATGGCAAAGACA \\
TAZ & \\
Forward & GTCACCAACAGTAGCTCAGATC \\
Reverse & AGTGATTACAGCCAGGTTAGAAAG \\
GAPDH & \\
Forward & CTTTGGTATCGTGGAAGGACTC \\
Reverse & GTAGAGGCAGGGATGATGTTCT
\end{tabular}

YAP, Yes-associated protein; TAZ, transcriptional co-activator with PDZ-binding motif.

Cell proliferation assay. An MTT assay was performed to measure the cell proliferation. After transfection, LOVO cells in the logarithmic growth phase were harvested with $0.25 \%$ trypsin (C0201; Beyotime Institute of Biotechnology) and seeded at a density of $5 \times 10^{3}$ cells per well in a 96-well plate. A total of $10 \mu \mathrm{l}$ MTT (Sigma-Aldrich; Merck KGaA, Darmstadt, Germany) was added to the cell culture medium of wells from designated groups every $24 \mathrm{~h}$. The plates were returned to the incubator for an additional $4 \mathrm{~h}$ at $37^{\circ} \mathrm{C}$ in a humidified atmosphere of $5 \% \mathrm{CO}_{2}$. Susbequently, the medium was removed and the cells were solubilized in $100 \mu \mathrm{l}$ dimethylsulfoxide. The absorbance was measured at $490 \mathrm{~nm}$ by using a spectrophotometer. Each experiment was repeated three times.

Apoptosis assay. For detection of cell apoptosis, an Annexin V-FITC apoptosis detection kit (A211-02; Vazyme Biotech Co., Ltd., Nanjing, China) was used. After $24 \mathrm{~h}$ of transfection, LOVO cells were washed with cold PBS and then labeled with Annexin V-fluorescein isothiocyanate and propidium iodide according to the manufacturer's instructions. BD FACSVerse flow cytometer (BD Biosciences, Franklin Lakes, NJ, USA) was applied to analyze cell apoptosis. All experiments were performed in triplicate.

Statistical analysis. Values are expressed as the mean \pm standard deviation. Student's t-test and one-way or two-way analysis of variance were used to assess differences between groups. $\mathrm{P}<0.05$ was considered to indicate a significant difference. All statistical analyses were performed using IBM SPSS Statistical software (version 20.0; IBM Corp., Armonk, NY, USA).

\section{Results}

YAP and TAZ expression in LOVO cells. A previous study reported that YAP and TAZ expression in LOVO cells was low (13). In the present study, the expression levels of YAP and TAZ were detected in LOVO cells, and the results were consistent with the previously reported ones (Fig. 1). To investigate the role of YAP and TAZ in LOVO cells, they were transfected with YAP and/or TZA expression plasmids. After 
A

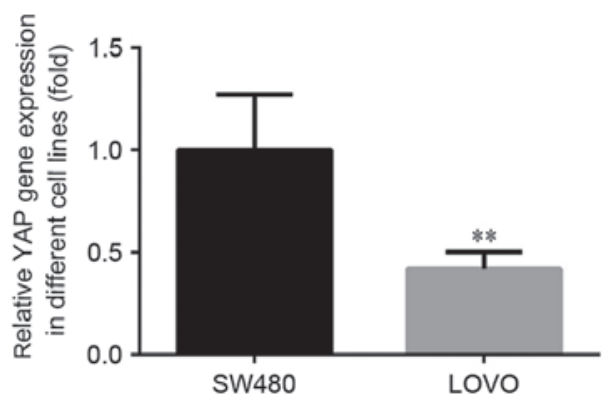

B

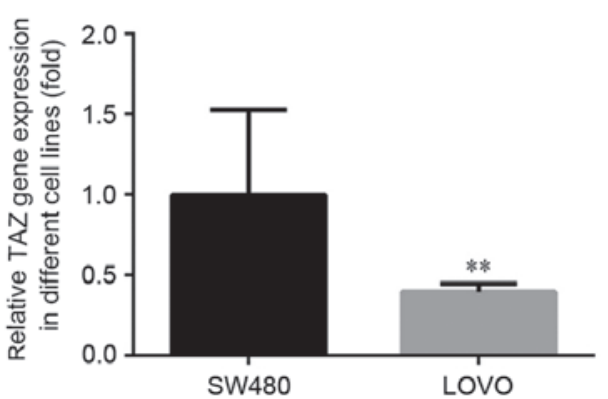

C

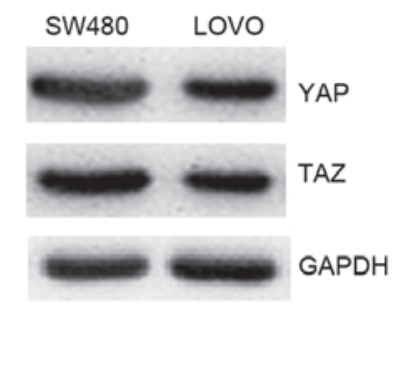

Figure 1. YAP and TAZ expression in LOVO cells. (A and B) mRNA and (C) protein expression levels of YAP and TAZ in SW480 and LOVO cells were determined by reverse-transcription quantitative polymerase chain reaction and western blot analysis, respectively. Values are expressed as the mean \pm standard deviation of three independent experiments. ${ }^{* *} \mathrm{P}<0.01$. YAP, Yes-associated protein; TAZ, transcriptional co-activator with PDZ-binding motif.

A

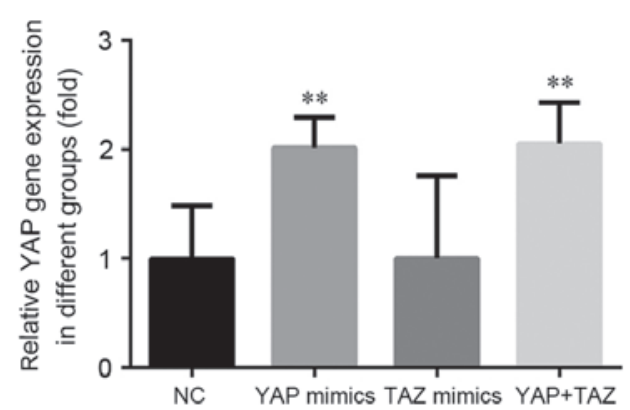

B

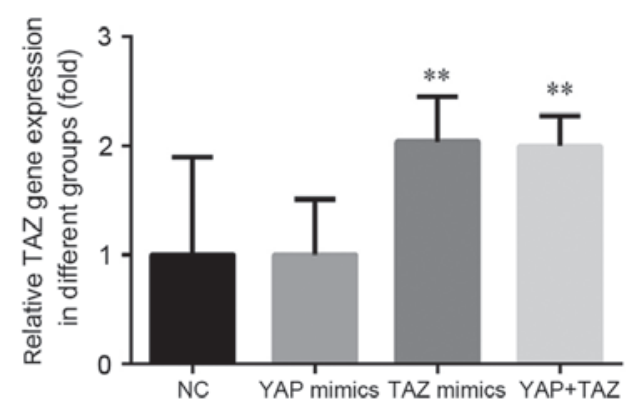

C

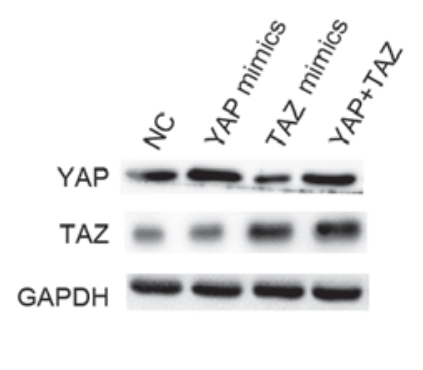

Figure 2. YAP and TAZ expression in transfected LOVO cells. After LOVO cells were transfected with YAP and/or TAZ plasmids for $24 \mathrm{~h}$, reversetranscription quantitative polymerase chain reaction and western blot analyses were performed to detect the (A and B) mRNA and (C) protein expression levels of YAP and TAZ. Values are expressed as the mean \pm standard deviation of three independent experiments. ${ }^{* *} \mathrm{P}<0.01$. YAP, Yes-associated protein; TAZ, transcriptional co-activator with PDZ-binding motif.

$24 \mathrm{~h}$ of transfection, the mRNA and protein expression levels of YAP and TAZ were determined by RT-qPCR and western blot analysis, respectively. The results suggested that YAP and/or TZA expression plasmids effectively enhanced YAP and/or TAZ expression in LOVO cells (Fig. 2).

Enhanced YAP and/or TAZ expression enhances the proliferation ability of LOVO cells. To investigate the effect of YAP and/or TAZ overexpression on LOVO cell proliferation, an MTT assay was performed. As demonstrated in Fig. 3, compared with that in the control, YAP or TAZ overexpression significantly increased the proliferation ability of LOVO cells, which was further increased as YAP and TAZ were co-overexpressed. These results indicated that either YAP or TAZ promoted LOVO cell proliferation, which was enhanced when YAP and TAZ were co-overexpressed.

Enhanced YAP and/or TAZ expression inhibits apoptosis of LOVO cells. Cell apoptosis was also measured by performing FCM after the respective treatments. As presented in Fig. 4, compared with that in the control group, the apoptotic rate of YAP- or TZA-overexpressing LOVO cells was significantly reduced, with YAP and TAZ co-overexpression resulting in an even greater reduction of the apoptotic rate.

Enhanced YAP and/or TAZ expression alters the expression of CTGF, Cyr61, Bcl2, Bax and caspase-3. CTGF and Cyr61

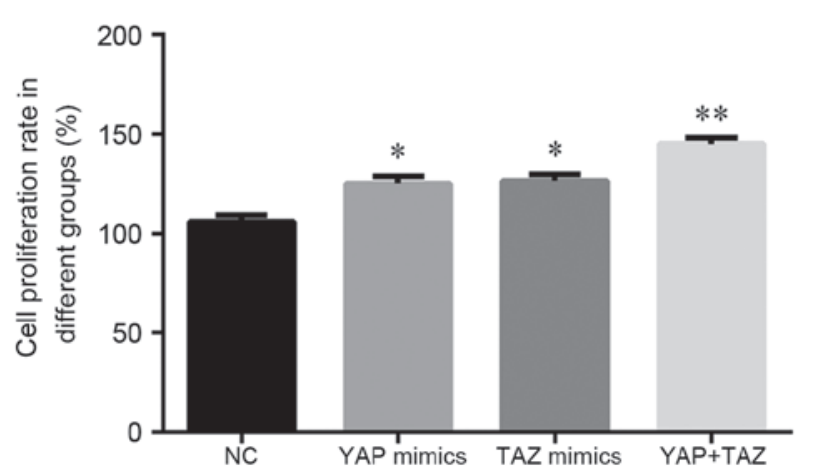

Figure 3. YAP and/or TAZ overexpression promotes the proliferation in LOVO cells. Cell proliferation was analyzed by an MTT assay at $24 \mathrm{~h}$ after LOVO cells were transfected with YAP and/or TAZ plasmids. Groups: NC, negative control; YAP mimics, cells transfected with YAP expression plasmids; TAZ mimics, cells transfected with TZA expression plasmids; YAP+TAZ, cells co-transfected with YAP and TAZ plasmids. Values are expressed as the mean \pm standard deviation of three independent experiments. ${ }^{*} \mathrm{P}<0.05,{ }^{* *} \mathrm{P}<0.01$. YAP, Yes-associated protein; TAZ, transcriptional co-activator with PDZ-binding motif.

are downstream genes of YAP and are regulated by YAP proteins. CTGF and Cyr61 were reported to have critical roles in improving cell proliferation, migration and invasion in cancer (15). In the present study, after YAP or TAZ was overexpressed, the protein expression levels of CTGF and Cyr61 were significantly higher compared with those in the control 

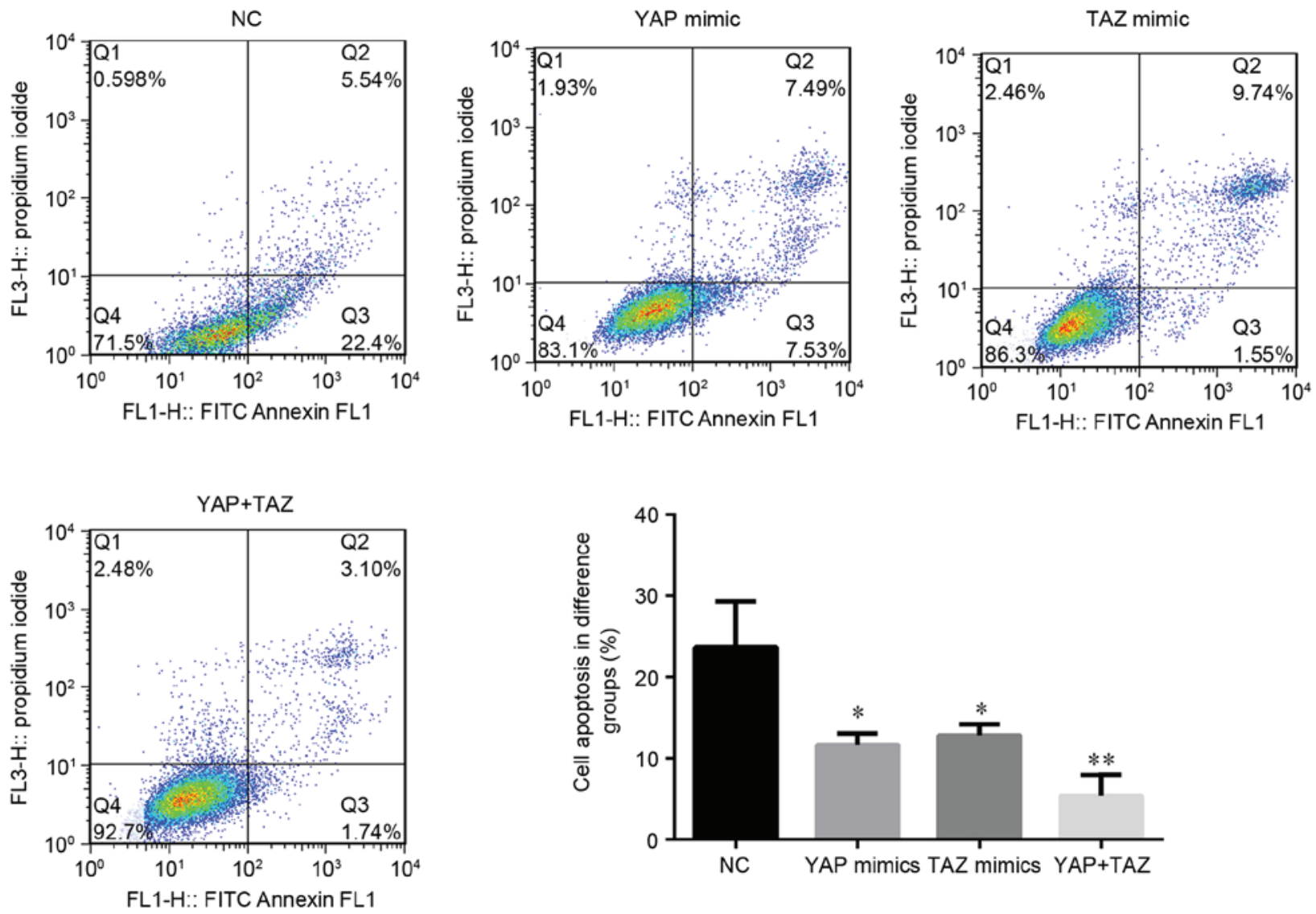

Figure 4. YAP and/or TAZ over-expression reduces the apoptosis of LOVO cells. After LOVO cells were transfected with YAP and/or TAZ plasmids for $24 \mathrm{~h}$, flow cytometry was performed for cell apoptosis detection. Groups: NC, negative control; YAP mimics, cells transfected with YAP expression plasmids; TAZ mimics, cells transfected with TZA expression plasmids; YAP+TAZ, cells co-transfected with YAP and TAZ plasmids. Values are expressed as the mean \pm standard deviation of three independent experiments. ${ }^{*} \mathrm{P}<0.05,{ }^{* *} \mathrm{P}<0.01$. YAP, Yes-associated protein; TAZ, transcriptional co-activator with PDZ-binding motif; FITC, fluorescein isothiocyanate; Q, quadrant.

group (Fig. 5). Of note, co-overexpression of YAP and TAZ further enhanced CTGF and Cyr61 expression.

In addition, to further explore the mechanisms of the reduction of cell apoptosis, the expression levels of the apoptosis-associated proteins Bax, Bcl-2 and caspase- 3 were determined by western blot analysis. The results indicated that the Bcl-2/Bax ratio of LOVO cells was markedly increased when YAP or TAZ was overexpressed, and as expected, the caspase-3 expression levels were notably inhibited (Fig. 5). Furthermore, these effects were even greater when YAP and TAZ were co-overexpressed.

\section{Discussion}

The present study demonstrated that YAP or TAZ overexpression significantly increased the proliferation ability of LOVO human colon cancer cells, while cell apoptosis was markedly inhibited. Co-overexpression of YAP and TAZ further promoted these effects on LOVO cell growth and apoptosis. In addition, tumor-associated genes (CTGF, Cyr61, Bcl-2, Bax and caspase-3) were differentially expressed in LOVO cells when YAP and/or TAZ was overexpressed. YAP and TAZ were found to act as oncogenes in human colon cancer and had complementary effects in regulating cell growth.

Cancer development is a complex process involving the interaction of multiple signaling pathways that regulate normal

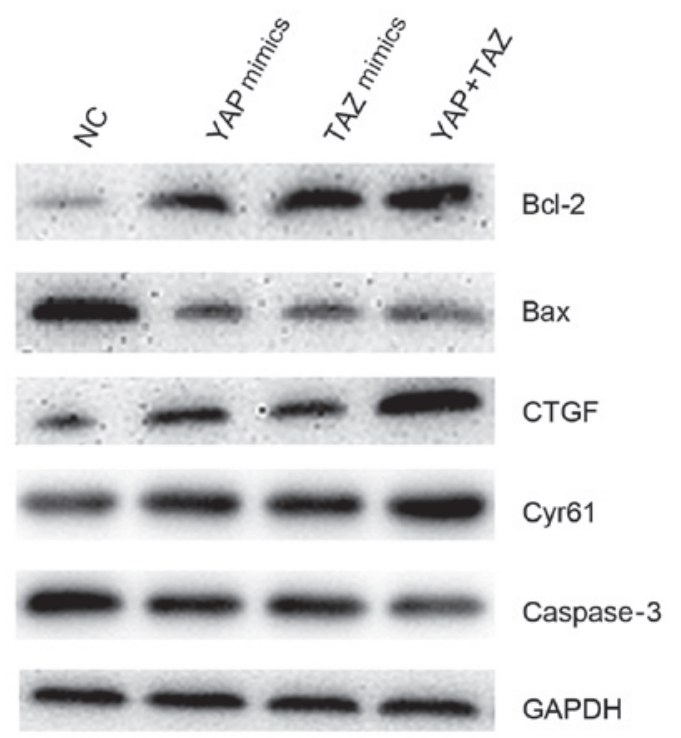

Figure 5. YAP and/or TAZ overexpression changes the protein expression of tumor- or apoptosis-associated genes in LOVO cells. At $24 \mathrm{~h}$ after the transfection, CTGF, Cyr61, Bcl-2, Bax and caspase-3 protein expression levels were determined by using western blot analysis. Groups: NC, negative control; YAP mimics, cells transfected with YAP expression plasmids; TAZ mimics, cells transfected with TZA expression plasmids; YAP+TAZ, cells co-transfected with YAP and TAZ plasmids. YAP, Yes-associated protein; TAZ, transcriptional co-activator with PDZ-binding motif; CTGF, connective tissue growth factor; Cyr61, cysteine-rich angiogenic inducer 61; Bcl-2, B-cell lymphoma 2; Bax, Bcl-2-associated X protein. 
cell growth. The Hippo signaling pathway has important roles in regulating cell fate and tissue growth. Studies have revealed the deregulation of the Hippo pathway in various human cancer types, including lung, liver, prostate, colon, breast and gastrointestinal cancers (16-20), and that it was frequently associated with poor prognosis of patients (21-23). YAP and TAZ are major downstream target genes of Hippo. Numerous studies have indicated the oncogenic role of YAP and TAZ in several solid tumor types $(16,18,24,25)$. YAP overexpression has been reported to have important effects on cell proliferation, survival, evasion of apoptosis, invasion and migration $(26,27)$, and TAZ was found to promote cell proliferation and enhance cell invasion and migration (28). The present study first detected the expression levels of YAP and TAZ in the LOVO human colon cancer cell line and the results revealed a low expression of YAP and TAZ. The role of YAP and/or TAZ in LOVO cells was then investigated by YAP and/or TAZ overexpression via plasmid transfection. The cell proliferation ability was determined using MTT assay, which indicated that when YAP or TAZ was overexpressed, the proliferation ability of LOVO cells was significantly increased, and co-overexpression of YAP and TAZ amplified this effect. These findings are consistent with those of a previous study (13). At the same time, the expression of the tumor-associated proteins CTGF and Cyr61, which have critical roles in promoting cancer cell proliferation, migration and invasion, was found to be enhanced following overexpression YAP and/or TAZ in LOVO cells.

The effect of YAP and/or TAZ overexpression on cell apoptosis was also determined in the present study. The results indicated that YAP and/or TAZ overexpression has an anti-apoptotic effect. To further explore the mechanisms underlying this cell apoptosis inhibition, the expression of apoptosis-associated proteins (Bax, Bcl-2 and caspase-3) was determined. The results indicated that YAP or TAZ overexpression inhibited LOVO cell apoptosis via increasing the Bcl-2/Bax ratio and reducing caspase-3 expression. Of note, these effects were further enhanced when YAP and TAZ were co-overexpressed.

In conclusion, the results of the present study indicated that upregulation of YAP and TAZ significantly influenced the behavior of LOVO human colon carcinoma cells, suggesting that YAP and TAZ have oncogenic roles in colon cancer progression. YAP and TAZ may serve as potential targets in the treatment of colon cancer in the future.

\section{References}

1. Siegel R, Naishadham D and Jemal A: Cancer statistics, 2013. CA Cancer J Clin 63: 11-30, 2013.

2. Ku G, Tan IB, Yau T, Boku N, Laohavinij S, Cheng AL, Kang YK and de Lima Lopes G Jr: Management of colon cancer: Resource-stratified guidelines from the Asian Oncology Summit 2012. Lancet Oncol 13: e470-e481, 2012.

3. De Roock W, De Vriendt V, Normanno N, Ciardiello F and Tejpar S: KRAS, BRAF, PIK3CA, and PTEN mutations: Implications for targeted therapies in metastatic colorectal cancer. Lancet Oncol 12: 594-603, 2011.

4. Chan SW, Lim CJ, Chen L, Chong YF, Huang C, Song H and Hong W: The Hippo pathway in biological control and cancer development. J Cell Physiol 226: 928-939, 2011.

5. Zhao B, Li L, Lei Q and Guan KL: The Hippo-YAP pathway in organ size control and tumorigenesis: An updated version. Genes Dev 24: 862-874, 2010.
6. Pan D: The hippo signaling pathway in development and cancer. Dev Cell 19: 491-505, 2010.

7. Harvey K and Tapon N: The Salvador-Warts-Hippo pathway-an emerging tumour-suppressor network. Nat Rev Cancer 7: 182-191, 2007.

8. Zhou D, Zhang Y, Wu H, Barry E, Yin Y, Lawrence E, Dawson D, Willis JE, Markowitz SD, Camargo FD and Avruch J: Mst1 and Mst2 protein kinases restrain intestinal stem cell proliferation and colonic tumorigenesis by inhibition of Yes-associated protein (Yap) overabundance. Proc Natl Acad Sci USA 108: E1312-E1320, 2011.

9. Avruch J, Zhou D and Bardeesy N: YAP oncogene overexpression supercharges colon cancer proliferation. Cell Cycle 11: 1090-1096, 2012

10. Wang Y, Xie C, Li Q, Xu K and Wang E: Clinical and prognostic significance of Yes-associated protein in colorectal cancer. Tumour Biol 34: 2169-2174, 2013.

11. Harvey KF, Zhang X and Thomas DM: The Hippo pathway and human cancer. Nat Rev Cancer 13: 246-257, 2013.

12. Yuen HF, McCrudden CM, Huang YH, Tham JM, Zhang X, Zeng Q, Zhang SD and Hong W: TAZ expression as a prognostic indicator in colorectal cancer. PLoS One 8: e54211, 2013.

13. Wang L, Shi S, Guo Z, Zhang X, Han S, Yang A, Wen W and Zhu Q: Overexpression of YAP and TAZ is an independent predictor of prognosis in colorectal cancer and related to the proliferation and metastasis of colon cancer cells. PLoS One 8: e65539, 2013.

14. Wang Y, Dong Q, Zhang Q, Li Z, Wang E and Qiu X: Overexpression of yes-associated protein contributes to progression and poor prognosis of non-small-cell lung caner. Cancer Sci 101: 1279-1285, 2010.

15. Planque $\mathrm{N}$ and Perbal B: A structural approach to the role of CCN (CYR61/CTGF/NOV) proteins in tumourigenesis. Cancer Cell Int 3: 15, 2003.

16. Zhao B, Wei X, Li W, Udan RS, Yang Q, Kim J, Xie J, Ikenoue T, Yu J, Li L, et al: Inactivation of YAP oncoprotein by the Hippo pathway is involved in cell contact inhibition and tissue growth control. Genes Dev 21: 2747-2761, 2007.

17. Dong J, Feldmann G, Huang J, Wu S, Zhang N, Comerford SA, Gayyed MF, Anders RA, Maitra A and Pan D: Elucidation of a universal size-control mechanism in Drosophila and mammals. Cell 130: 1120-1133, 2007.

18. Steinhardt AA, Gayyed MF, Klein AP, Dong J, Maitra A, Pan D, Montgomery EA and Anders RA: Expression of Yes-associated protein in common solid tumors. Hum Pathol 39: 1582-1589, 2008.

19. Shi P, Feng J and Chen C: Hippo pathway in mammary gland development and breast cancer. Acta Biochim Biophys Sin (Shanghai) 47: 53-59, 2015.

20. Kang W, Cheng AS, Yu J and To KF: Emerging role of Hippo pathway in gastric and other gastrointestinal cancers. World J Gastroenterol 22: 1279-1288, 2016.

21. Zhang X, George J, Deb S, Degoutin JL, Takano EA, Fox SB; AOCS Study group, Bowtell DD and Harvey KF: The Hippo pathway transcriptional co-activator, YAP, is an ovarian cancer oncogene. Oncogene 30: 2810-2822, 2011

22. Hall CA, Wang R, Miao J, Oliva E, Shen X, Wheeler T, Hilsenbeck SG, Orsulic S and Goode S: Hippo pathway effector Yap is an ovarian cancer oncogene. Cancer Res 70: 8517-8525, 2010.

23. Xu MZ, Yao TJ, Lee NP, Ng IO, Chan YT, Zender L, Lowe SW, Poon RT and Luk JM: Yes-associated protein is an independent prognostic marker in hepatocellular carcinoma. Cancer 115: 4576-4585, 2009

24. Camargo FD, Gokhale S, Johnnidis JB, Fu D, Bell GW, Jaenisch R and Brummelkamp TR: YAP1 increases organ size and expands undifferentiated progenitor cells. Curr Biol 17: 2054-2060, 2007.

25. Wang X, Su L and Ou Q: Yes-associated protein promotes tumour development in luminal epithelial derived breast cancer. Eur J Cancer 48: 1227-1234, 2012.

26. Zhao B, Ye X, Yu J, Li L, Li W, Li S, Yu J, Lin JD, Wang CY, Chinnaiyan AM, et al: TEAD mediates YAP-dependent gene induction and growth control. Genes Dev 22: 1962-1971, 2008.

27. Hao Y, Chun A, Cheung K, Rashidi B and Yang X: Tumor suppressor LATS1 is a negative regulator of oncogene YAP. J Biol Chem 283: 5496-5509, 2008.

28. Chan SW, Lim CJ, Guo K, Ng CP, Lee I, Hunziker W, Zeng Q and Hong W: A role for TAZ in migration, invasion, and tumorigenesis of breast cancer cells. Cancer Res 68: 2592-2598, 2008. 